\title{
Recent advances in neuroimaging of bladder, bowel and sexual function
}

\author{
Rosa L. Coolen, Ilse M. Groenendijk, and Bertil F.M. Blok
}

\begin{abstract}
Purpose of review
In this review, we summarize recent advances in the understanding of the neural control of the bladder, bowel and sexual function, in both men and women.

\section{Recent findings}

Evidence of supraspinal areas controlling the storage of urine and micturition in animals, such as the pontine micturition centre, emerged in the early 20 th century. Neurological stimulation and lesion studies in humans provided additional indirect evidence for additional bladder-related brain areas. Thereafter, functional neuroimaging in humans with PET and FMRI provided more direct evidence of the involvement of these brain areas. The areas involved in the storage and expulsion of urine also seem to be involved in the central control of storage and expulsion of feces. Furthermore, most knowledge on the brain control of sexual function is obtained from dynamic imaging in human volunteers. Relatively little is known about the dysfunctional central circuits in patients with pelvic organ dysfunction.
\end{abstract}

\begin{abstract}
Summary
FMRI has been the most widely used functional neuroimaging technique in the last decade to study the central control of bladder function, anorectal function and sexual function. The studies described in this review show which sensory and motor areas are involved, including cortical and subcortical areas. We propose the existence of a switch-like phenomenon located in the pons controlling micturition, defecation and orgasm.
\end{abstract}

\section{Keywords}

defecation, FMRI, lower urinary tract, neuroimaging, sexual function

\section{INTRODUCTION}

Functional neuroimaging techniques include functional MRI (fMRI), positron emission tomography (PET), electroencephalography (EEG), magnetoencephalography (MEG), functional near infrared spectroscopy (fNIRS) and single-photon emission computed tomography (SPECT). These techniques detect changes in neuronal activity with a specific neuronal marker. Each functional neuroimaging modality has its pros and cons. Imaging at $7 \mathrm{~T}$ has a significantly higher functional sensitivity and spatial resolution compared to imaging at 3T, but at the expense of an increase in motion artifacts [1]. However, fMRI has a limited temporal resolution compared to EEG and NIRS [2]. Conversely, EEG and fNIRS can only detect cortical activity and, therefore, have a lower spatial resolution than fMRI. The present review focusses on functional neuroimaging literature on the bladder, bowel and sexual function. There exists a remarkable overlap in central activation in bladder, anorectal and sexual control. Micturition, defecation and orgasm are switch-like phenomena, which are controlled by supraspinal structures. We refer to the pelvic floor muscles involved in these functions as the pelvic closure muscles, these include the urethral and anal sphincters. The pelvic organs of which we describe the neural control are the bladder, the anorectum and the internal and external genitalia.

\section{Bladder function}

The lower urinary tract (LUT) has two functions: storage of urine and micturition, which both require coordinated neural control by the brainstem. Evidence from studies in cats of the supraspinal areas

Department of Urology, Erasmus Medical Center, Rotterdam, The Netherlands

Correspondence to Rosa L. Coolen, Department of Urology, Wytemaweg 80, 3015 CN Rotterdam, The Netherlands. Tel: +31 10703 2414; fax: +31 10703 5632; e-mail: r.coolen@erasmusmc.nl

Curr Opin Urol 2020, 30:480-485

DOI:10.1097/MOU.0000000000000772 


\section{KEY POINTS}

- FMRI is the functional neuroimaging technique most widely used to study the central control urinary bladder function, anorectal function and sexual function.

- fMRI is usually chosen as neuroimaging technique because of its relatively high spatial resolution compared to other functional neuroimaging techniques.

- We propose the existence of a switch-like mechanism originating in the pons controlling the opening of the pelvic closure muscles during micturition, defecation and orgasm.

- We propose that suprapontine sensory, motor and limbic brain areas modulate final common pathway areas in the pons and spinal cord and, therefore, influence both pelvic organ and pelvic closure muscle functions.

controlling the storage of urine and micturition, such as the pontine micturition centre (PMC), emerged in the early 20th century and was confirmed by additional animal studies $\left[3^{*}, 4^{\prime \prime}\right]$. Subsequently, functional neuroimaging studies concerning LUT control in humans became available. A study, using PET, showed that the periaqueductal grey (PAG) and the PMC [also called Barrington's nucleus or the M(edial)-region, later in this article referred to as the pelvic motor centre] are involved, and the authors proposed that the PMC functions as a switch that initiates micturition $\left[5^{\boldsymbol{\prime}}, 6,7^{\boldsymbol{\prime}}\right]$. The pontine storage or continence centre [PSC, also called L(ateral)-region], later in this article referred to as the pelvic storage centre, is postulated to be responsible for maintaining continence inbetween voids [8]. The peripheral nerves innervating the lower urinary tract are the hypogastric, the pelvic, and the pudendal nerves. These nerves, together with local connections within the spinal cord, ensure necessary reflexes of the bladder and pelvic floor muscle tone in response to changes in bladder filling. Control of the micturition reflex pathways by suprapontine brain areas ensures micturition under the appropriate circumstances [9].

\section{Bowel function}

The rectum serves as a reservoir for stool produced by the small and large intestines. The anus contains an internal and external sphincter ensuring faecal continence. Faecal continence and defecation are partly controlled by the same brain structures as micturition [10]. The spinal and supraspinal brain areas involved in anorectal function are less often studied than those involved in bladder function.
The descending control of the external anal and urethral sphincters comprises a common pathway in the brainstem, the spinal cord and the pudendal nerve [11]. There is evidence supporting the concept that the PMC controls the distal colon, rectum and internal anal sphincter [12-14]. Furthermore, the hypothesis is that the external anal sphincter is controlled by the PSC, which ensures faecal continence. The peripheral nerves innervating the anorectum and the external anal sphincter are the hypogastric, the pelvic and the pudendal nerves. The recent advances in the understanding of the central neural control of anorectal function and the similarities to the control of bladder function are discussed in this review.

\section{Sexual function}

The internal and external genitalia serve as organs important for sexual function aimed at pleasure and reproduction. The first evidence of the central areas controlling sexual function came from experiments in cats and monkeys. Electrical stimulation in monkeys revealed that several brain areas of the limbic system are involved in penile erection: the hippocampus, anterior and medial parts of the thalamus, the hypothalamus, the mammillary bodies, the frontal lobe and the cingulate gyrus [15]. More recent studies used a neuronal tracer injected in the penis or clitoris in rats. These experiments revealed a possible role for the nucleus paragigantocellularis and the PMC in the brainstem in penile function and in clitoral function $[16,17]$. A lesion of the nucleus paragigantocellularis abolishes inhibition of erection and ejaculation [18]. Furthermore, electrical stimulation of the paraventricular nucleus of the hypothalamus induced penile erection and ejaculation in rats [19]. These animal studies implicate a function for higher brain areas in sexual function in conjunction with diencephalon, midbrain and brainstem areas.

\section{NEUROIMAGING OF BRAIN AREAS INVOLVED IN THE STORAGE OF URINE AND MICTURITION}

In the following two paragraphs, we first describe the central control of the urinary bladder and, thereafter, the voluntary control of pelvic floor muscle tone with regard to micturition.

\section{Bladder}

The bladder serves as a reservoir for urine. When the amount of urine in the bladder exceeds a certain individual threshold, an urge to void may arise. If the circumstances are appropriate, micturition can 
start. A 3T fMRI study in healthy male and female individuals investigating brain activity during bladder filling confirmed and extended the previous PET studies with activation of the dorsolateral prefrontal cortex, the orbitofrontal/frontopolar cortex, the ventrolateral prefrontal cortex, the anterior cingulate cortex, the left insula, the right insula, the hypothalamus and the left basal ganglia [20]. In both healthy male and female volunteers it was demonstrated using fMRI that at the end of the storage phase, when the bladder was filled and there was a strong desire to void, the prefrontal cortex, the anterior cingulate cortex, the hypothalamus, the temporal lobes and the left caudate nucleus were active [21]. The PAG, the pons, the insula, the thalamus, the prefrontal cortex, the parietal operculum and the cingulate cortex are active during the transition from urine storage to micturition [22]. The insula, parts of the prefrontal cortex, the cingulate cortex and the hypothalamus are so-called limbic brain structures involved in the processing of sensing the interior and exterior of the individual and translate this information to behaviour important for vital functions. The basal ganglia are considered to be involved in processing and modulation of motor activity. Arya et al. [23"] performed a meta-analysis of neuroimaging studies on bladder filling using activation likelihood estimation, which showed significant activation of the thalamus, the insula, the PAG and the cerebellum during bladder filling.

\section{Pelvic floor}

Voluntary and involuntary pelvic floor muscle contractions are controlled by different brain areas. A $3 \mathrm{~T}$ fMRI study during voluntary pelvic floor muscle contraction in both healthy males and females revealed activation in the primary motor cortex and the supplementary motor area [24]. A 7T fMRI study in individual healthy male subjects showed activation in the primary motor cortex, the supplementary motor area, the midcingulate gyrus, the insula, the putamen, the thalamus and the anterior and posterior lobes of the cerebellum during pelvic floor muscle contraction [25]. Involuntary contraction of the pelvic floor musculature depends on the PSC and is a brainstem and spinal cord mediated reflex, continuously activated, which ensures continence during the storage of urine.

\section{NEUROIMAGING OF BRAIN AREAS INVOLVED IN ANORECTAL FUNCTION}

In the following two paragraphs we describe the neuroimaging of anorectal function during rectal distension and anal sphincter contractions.

\section{Rectum}

Rectal distension was studied in healthy volunteers using both $1.5 \mathrm{~T}$ and $3 \mathrm{~T}$ fMRI. The 3T fMRI study demonstrated activation during rectal distension in the right mid-insula, the bilateral inferior parietal lobe and the right operculum [26]. Activation was found, using 1.5T fMRI, in the anterior cingulate gyrus, the insula, the thalamus and the supplementary motor area bilaterally [27]. This coincided with prefrontal deactivation. Increasing rectal distension to a level at which it was painful showed increased activity in the aforementioned brain areas without activation of any additional brain areas. A meta-analysis was conducted by Halani et al. [28"] showing that activation partly overlaps during bladder filling and rectal distension. The right insula and the right and left thalamus were activated during bladder filling and rectal distension. Combining the evidence from animal studies locating the pontine defecation centre in the PMC, the overlap of central micturition and defecation control becomes more evident. There is no evidence of distinct neurons within the PMC involved in either bladder control or rectal control [29]. Thus, it seems that neurons in the PMC are involved in both functions. To completely understand the neural control of anorectal function, the act of defecation should be studied.

\section{Pelvic floor}

A 1.5T fMRI study in healthy volunteers showed activation of the primary motor cortex and the supplementary motor area during external anal sphincter contractions [27]. Furthermore, activation was present in the anterior and medial insula, the primary somatosensory and the secondary somatosensory cortex bilaterally. Another 1.5T fMRI study showed activation of sensory areas, motor areas, the cingulate gyrus, the prefrontal cortex, the insula and the parietooccipital cortex [30]. The total volume of cortical activity was higher when the volunteers exerted maximal effort, which was monitored by measuring pressure in the anal canal using a catheter, whereby the subjects were instructed to contract their anal sphincter both maximally and submaximally. Both of the aforementioned studies describe the voluntary, but not the involuntary, control of anal sphincter muscle tone. In comparison to the 7T fMRI study mentioned earlier in the section about pelvic floor muscle tone in relation to micturition, we see that the activated brain areas are partly the same [25]. However, the 7T fMRI study revealed additional activation in the cerebellum, putamen and the thalamus which was not shown in the aforementioned 1.5T fMRI studies on anal sphincter contractions. 
NEUROIMAGING OF BRAIN AREAS INVOLVED IN SEXUAL FUNCTION IN WOMEN

A description of neuroimaging of sensory functions of the female genitalia and neuroimaging of female orgasm is given in the following two paragraphs.

\section{Internal and external genitalia}

Studies on the sensory representation of the genitalia in women used either tactile stimulation or transcutaneous electrical stimulation. A 3T fMRI study was performed to map the sensory representation of the vagina and cervix on the sensory cortex [31]. Healthy right-handed women performed clitoral, vaginal and cervical self-stimulation, which all showed activation of the medial paracentral lobule in the sensory cortex. The activated areas were partly overlapping but not completely, implicating separate representations of the clitoris, vagina and cervix in the sensory cortex. An fMRI study by Wise et al. [32] compared tactile self-stimulation of the clitoris to imagined tactile stimulation of the clitoris. Tactile self-stimulation of the clitoris resulted in activation of the paracentral lobule, the right parietal operculum, the frontal pole, the orbitofrontal cortex, the cerebellum, the hand region of the primary somatosensory cortex and the premotor cortex. Imagined tactile stimulation of the clitoris activated the same areas and, additionally, the left parietal operculum, the insular cortex, the amygdala, the hippocampus and the inferior parietal lobule. These results are in line with previous descriptions locating the sensory representation of the clitoris in the paracentral lobule [31]. On the contrary, another 3T fMRI study located the sensory representation of the clitoris on the convexity of the postcentral gyrus [33]. fMRI scanning with increased magnetic field strength might resolve these inconsistent findings in the future.

\section{Female orgasm}

An fMRI study revealed activation of multiple brain areas leading up to orgasm, followed by a decrease in activity in the same areas, thereafter. Examples of these areas are: the nucleus accumbens, the insula, the anterior cingulate cortex, the orbitofrontal cortex, the operculum, the right angular gyrus, the paracentral lobule, the cerebellum, the hippocampus, the amygdala, the hypothalamus, the ventral tegmental area and the dorsal raphe [34]. This study compared self-stimulation to partner induced stimulation. The two conditions showed similar activation patterns during orgasm. However, there was a difference in the level of activity during self-stimulation compared to partner-induced stimulation, showing more activity mid-stimulation and less activity during late stimulation in the self-stimulation condition.

\section{NEUROIMAGING OF BRAIN AREAS INVOLVED IN SEXUAL FUNCTION IN MEN}

Firstly, we describe neuroimaging of the internal and external genitalia in men and, thereafter, we describe recent advances in neuroimaging of sexual function in men.

\section{Internal and external genitalia}

No neuroimaging studies in healthy volunteers about the neural control of the internal genitalia in men, which are the prostate and the seminal vesicles, were identified. A study using a retrograde transneuronal tracer injected in the prostate of rats, revealed labelling predominantly in the ventrolateral medulla, the medullary raphe, the PMC and the paraventricular nucleus of the hypothalamus [35]. The external genitalia in men comprise the penis and the scrotum. A study in 2005 revealed that the primary sensory representation of the penis lies on the convexity of the postcentral gyrus in an area that overlaps with the sensory representation of the abdominal wall [36"']. Recently this result was confirmed in single subjects by tactile stimulation of the penile shaft during 7T fMRI scanning [37]. This is in contrast to earlier descriptions in which the external male genital region was located on the medial wall in the interhemispheric fissure.

\section{Male orgasm}

An fMRI study of healthy male individuals compared visual sexual stimuli and visual nonsexual stimuli [38]. Increased activation was observed after presentation of visual sexual stimuli compared to visual nonsexual stimuli in the bilateral medial occipital gyrus, the fusiform gyrus, the praecuneus, the inferior parietal cortex, the orbital frontal cortex, the thalamus, the insula, the globus pallidus, the putamen, and the amygdala. A study was conducted in healthy male subjects using NIRS to see whether this technique is capable of detecting prefrontal cortical changes in blood flow as a response to visual sexual stimulation [39]. This study showed alterations in oxygenated and deoxygenated haemoglobin in the prefrontal cortex in response to visual sexual stimuli compared to visual nonsexual stimuli. The studies described here do not show which brain areas are involved in the male orgasm, they merely describe which brain areas are involved in arousal. 


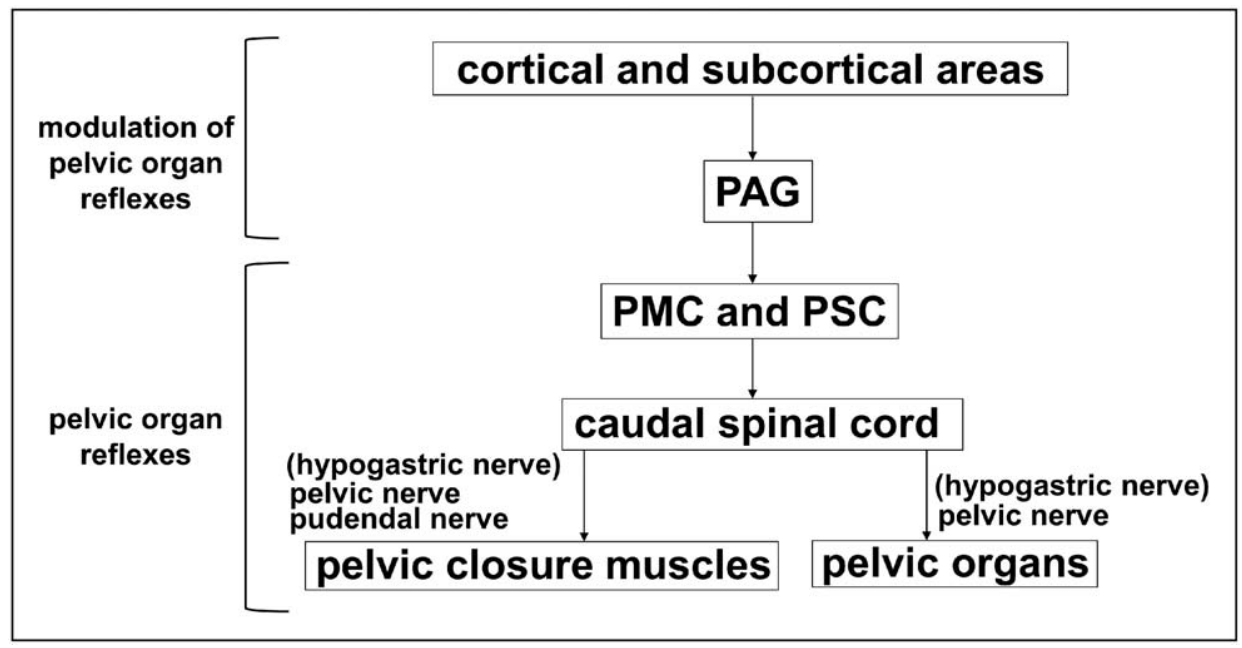

FIGURE 1. Neural control of the pelvic organs. Overview of the central control of the pelvic organs and the pelvic closure muscles in both women and men. Suprapontine brain areas modulate the core pathway originating in the pons, exerting its effect through the spinal cord and the peripheral nerves innervating the pelvic organs and the pelvic floor closure muscles, which are part of the pelvic floor (internal and external urethral sphincters, internal and external anal sphincters, $\mathrm{m}$. puborectalis, m. ischiocavernosus, m. bulbospongiosus). PAG, periaqueductal grey; PMC, pelvic motor centre (also referred to as the pontine micturition centre); PSC, pelvic storage centre (also called pontine storage centre or pontine continence centre).

\section{CONCLUSION}

Bladder, bowel (or more specific, anorectal) and sexual functioning is controlled by central components originating in the pons, specifically in the pelvic motor centre (also referred to as the PMC) and the pelvic storage centre (PSC, Fig. 1). We propose a switch-like mechanism located in the pelvic motor centre, of which the activity is modulated by suprapontine brain areas, controlling micturition, defecation and orgasm. These suprapontine brain areas are proposed to modulate the control of the pelvic organs, including the bladder, anorectum and pelvic closure muscles, including the urethral and anal sphincters. Pelvic organ function is modulated by several common brain areas including the insula, the cingulate gyrus and the thalamus. Voluntary control of pelvic closure muscle contractions for the control of micturition and anorectal function shows common activation in the primary motor cortex, the cingulate gyrus and the insula. The neural control of the pelvic closure muscles in continuous storage and sexual function is involuntary, and therefore, other brain areas are activated. fMRI studies have provided many insights, however, further research into the interaction between the different involved brain areas is necessary.

\section{Acknowledgements}

None.

\section{Financial support and sponsorship}

B.B. was supported by grants from Zorg Onderzoek Nederland en Medische Wetenschappen (ZonMw), Erasmus Medical Center Efficiency Research Grant and Stichting Urologisch Wetenschappelijk Onderzoek (SUWO).

\section{Conflicts of interest}

There are no conflicts of interest.

\section{REFERENCES AND RECOMMENDED}

\section{READING}

Papers of particular interest, published within the annual period of review, have been highlighted as:

- of special interest

- of outstanding interest

1. Beisteiner $R$, Robinson $S$, Wurnig $M$, et al. Clinical $f M R I$ : evidence for a $7 T$ benefit over 3T. Neuroimage 2011; 57:1015-1021.

2. Detre JA, Wang J. Technical aspects and utility of fMRI using BOLD and ASL. Clin Neurophysiol 2002; 113:621-634.

3. Barrington FJF. The effect of lesions of the hind-and mid-brain on micturition

- in the cat. Quart J Exp Physiol 1925; 15:81-102.

Demonstrated that the PMC controls micturition in cats.

4. Holstege G, Griffiths D, de Wall H, Dalm E. Anatomical and physiological

- observations on supraspinal control of bladder and urethral sphincter muscles in the cat. J Comp Neurol 1986; 250:449-461.

Demonstrated spinal projections from the PMC and PSC in cats. Furthermore, electrical stimulation of the PMC decreased urethral pressure and electrical stimulation of the PSC increased urethral pressure.

5. Blok BFM, Willemsen AT, Holstege G. A PET study on brain control of -1. micturition in humans. Brain 1997; 120(Pt 1):111-121.

Demonstrated the existence and location of the human PMC and PSC.

6. Blok BF, Holstege G. Ultrastructural evidence for a direct pathway from the pontine micturition center to the parasympathetic preganglionic motoneurons of the bladder of the cat. Neurosci Lett 1997; 222: $195-198$ 
7. Blok BF, Holstege G. Direct projections from the periaqueductal gray to the

- pontine micturition center (M-region). An anterograde and retrograde tracing study in the cat. Neurosci Lett 1994; 166:93-96.

Demonstrated the direct projection from the PAG to the PMC

8. Blok BF, Holstege G. Two pontine micturition centers in the cat are not interconnected directly: implications for the central organization of micturition. J Comp Neurol 1999; 403:209-218.

9. Blok BF, Sturms LM, Holstege G. A PET study on cortical and subcortical control of pelvic floor musculature in women. J Comp Neurol 1997; 389:535-544

10. Bishop B, Garry RC, Roberts TDM, Todd JK. Control of the external sphincter of the anus in the cat. J Physiol 1956; 134:229.

11. Mackel R. Segmental and descending control of the external urethral and anal sphincters in the cat. J Physiol 1979; 294:105-122.

12. Holstege G, Tan J. Supraspinal control of motoneurons innervating the striated muscles of the pelvic floor including urethral and anal sphincters in the cat. Brain 1987; 110:1323-1344.

13. Pavcovich LA, Yang M, Miselis RR, Valentino RJ. Novel role for the pontine micturition center, Barrington's nucleus: evidence for coordination of colonic and forebrain activity. Brain Res 1998; 784(1-2):355-361.

14. Rouzade-Dominguez ML, Pernar L, Beck S, Valentino RJ. Convergent responses of Barrington's nucleus neurons to pelvic visceral stimuli in the rat: a juxtacellular labelling study. Eur J Neurosci 2003; 18:3325-3334.

15. MacLean PD, Ploog DW. Cerebral representation of penile erection. J Neurophysiol 1962; 25:29-55.

16. Marson L, Platt KB, McKenna KE. Central nervous system innervation of the penis as revealed by the transneuronal transport of pseudorabies virus. Neuroscience 1993; 55:263-280.

17. Marson L. Central nervous system neurons identified after injection of pseudorabies virus into the rat clitoris. Neurosci Lett 1995; 190:41-44.

18. Marson L, McKenna KE. The identification of a brainstem site controlling spinal sexual reflexes in male rats. Brain Res 1990; 515:303-308.

19. Chen KK, Chan SH, Chang LS, Chan JY. Participation of paraventricular nucleus of hypothalamus in central regulation of penile erection in the rat. $J$ Urol 1997; 158:238-244.

20. Walter M, Leitner L, Michels L, et al. Reliability of supraspinal correlates to lower urinary tract stimulation in healthy participants - A fMRI study. NeuroImage 2019; 191:481-492.

21. Gao $Y$, Liao L, Blok BFM. A resting-state functional MRI study on central control of storage: brain response provoked by strong desire to void. Int Urol Nephrol 2015; 47:927-935.

22. Michels L, Blok BFM, Gregorini F, et al. Supraspinal control of urine storage and micturition in men-an fMRI study. Cereb Cortex 2015; 25:3369-3380.

23. Arya NG, Weissbart SJ, Xu S, Rao H. Brain activation in response to bladder

- filling in healthy adults: an activation likelihood estimation meta-analysis of neuroimaging studies. Neurourol Urodyn 2017; 36:960-965.

Demonstrated activation of the thalamus, the insula, the PAG and the cerebellum during bladder filling using activation likelihood estimation.
24. Yani MS, Wondolowski JH, Eckel SP, et al. Distributed representation of pelvic floor muscles in human motor cortex. Sci Rep 2018; 8:7213.

25. Groenendijk IM, Luijten SPR, de Zeeuw $\mathrm{Cl}$, et al. Whole brain 7T-fMRI during pelvic floor muscle contraction in male subjects. Neurourol Urodyn 2019; 39:382-392.

26. Rubio A, Van Oudenhove L, Pellissier S, et al. Uncertainty in anticipation of uncomfortable rectal distension is modulated by the autonomic nervous system-a fMRI study in healthy volunteers. Neuroimage 2015; 107:10-22.

27. Bittorf $B$, Ringler $R$, Forster $C$, et al. Cerebral representation of the anorectum using functional magnetic resonance imaging. Br J Surg 2006; 93:1251-1257.

28. Halani PK, Andy UU, Rao H, Arya LA. Regions of the brain activated in bladder

- filling vs rectal distention in healthy adults: A meta-analysis of neuroimaging studies. Neurourol Urodyn 2019; 39:58-65.

Demonstrated overlap in activation during bladder filling and rectal distention. The right insula and the right and left thalamus were activated during bladder filling and rectal distention.

29. Vizzard MA, Brisson M, de Groat WC. Transneuronal labeling of neurons in the adult rat central nervous system following inoculation of pseudorabies virus into the colon. Cell Tissue Res 2000; 299:9-26.

30. Kern MK, Arndorfer RC, Hyde JS, Shaker R. Cerebral cortical representation of external anal sphincter contraction: effect of effort. Am J Physiol Gastrointest Liver Physiol 2004; 286:G304-G311.

31. Komisaruk BR, Wise $N$, Frangos $E$, et al. Women's clitoris, vagina, and cervix mapped on the sensory cortex: fMRI evidence. J Sex Med 2011; $8: 2822-2830$

32. Wise NJ, Frangos E, Komisaruk BR. Activation of sensory cortex by imagined genital stimulation: an fMRI analysis. Socioaffect Neurosci Psychol 2016; 6:31481.

33. Michels L, Mehnert U, Boy S, et al. The somatosensory representation of the human clitoris: an fMRI study. Neuroimage 2010; 49:177-184.

34. Wise NJ, Frangos E, Komisaruk BR. Brain activity unique to orgasm in women: an fMRI analysis. J Sex Med 2017; 14:1380-1391.

35. Orr R, Marson L. Identification of CNS neurons innervating the rat prostate: a transneuronal tracing study using pseudorabies virus. J Autonom Nerv Syst 1998; 72:4-15.

36. Kell CA, von Kriegstein $\mathrm{K}$, Rosler A, et al. The sensory cortical representation

-1. of the human penis: revisiting somatotopy in the male homunculus. J Neurosci 2005; 25:5984-5987.

3T fMRI study on the processing of penile sensation in the primary sensory cortex.

37. Luijten SPR, Groenendijk IM, Holstege JC, et al. Single subject and group whole-brain fMRI mapping of male genital sensation at 7 Tesla. Sci Rep 2020; 10:2487.

38. Seok JW, Sohn JH, Cheong C. Neural substrates of sexual arousal in heterosexual males: event-related fMRI investigation. J Physiol Anthropol $2016 ; 35: 8$.

39. Kim E, Kim S, Zephaniah PV, et al. Simultaneous Monitoring of hemodynamic response in the pre-frontal cortex and genital organ during sexual arousal using near-infrared spectroscopy. Sex Med 2018; 6:234-238. 